\title{
BIOADHESIVE MULTIPARTICULATE (MICROSPHERS) DRUG DELIVERY SYSTEM: A REVIEW
}

\author{
*Ravindra B. Borade ${ }^{1}$, Yogesh Y. Mahajan ${ }^{1}$, Nilesh P. Jadhav ${ }^{1}$, Jaiprakash V. Kokane ${ }^{1}$, Md. Rageeb Md. Usman², \\ Mrugendra B. Potdar ${ }^{3}$ \\ ${ }^{1}$ S. N. D. College of Pharmacy, Yeola, Dist Nasik, Maharashtra, India \\ ${ }^{2}$ Assistant Professor, Department of Pharmacognosy, Smt. S. S. Patil College of Pharmacy, Chopda, Maharashtra, India \\ ${ }^{3}$ Assistant Professor, Department of Pharmaceutics, Smt. S. S. Patil College of Pharmacy, Chopda, Maharashtra, India \\ *Corresponding author: E mails: ravindraborade0@ gmail.com, Ph. No.:07387036963
}

\begin{abstract}
The concept of controlled drug delivery has been traditionally used to obtain specific release rates or targeting of active ingredients. The phenomenon of bioadhesion has been studied extensively in the last decade and applied to improve the performance of these drug delivery systems. Recent advances in polymer science and drug carrier technologies have promulgated the development of novel drug carriers such as bioadhesive microspheres that have boosted the use of "bioadhesion" in drug delivery. This article presents the spectrum of potential applications of bioadhesive microspheres in controlled drug delivery ranging from the small molecules, to peptides, and to the macromolecular drugs such as proteins, oligonucleotides and even DNA. The development of mucus or cell-specific bioadhesive polymers and the concepts of cytoadhesion and bioinvasion provide unprecedented opportunities for targeting drugs to specific cells or intracellular compartments. Developments in the techniques for in vitro and in vivo evaluation of bioadhesive microspheres have also been discussed.

Keywords: - Bioadhesion, Bioadhesive Microspheres, Development, Polymers.
\end{abstract}

\section{INTRODUCTION}

\section{Microsphere ${ }^{l}$}

Recently, insoluble drug carriers for prolonged and controlled delivery of therapeutic agents in biological system have generated interest. A microsphere is defined as "a monolithic structure of the drug or therapeutic agent distributed throughout the matrix either as a molecular dispersion or as a dispersion of particles, in the size range 1-500 $\mu \mathrm{m} . "$

\section{Production Technology of Microsphere}

\section{Polymer Phase-Separation Method}

The general outline of the process consists of three steps carried out under continuous agitation, they are:

a) Formation of three immiscible chemical phases.

b) Deposition of polymer coat upon the core material by controlled physical mixing of coating material (while liquid) and the core material in the manufacturing vehicle.

c) The next process involves rigidisation of the coating, usually by thermal, cross linking or desolvation techniques.

\section{Emulsification Method (Emulsification Solvent Evaporation/ Emulsification Solvent Extraction)}

This technique is based on the evaporation of the internal phase of an emulsion by agitation. The formation of solid microspheres is brought about by the evaporation of volatile solvent at the interface between continuous phase and air, this cause partitioning across the interface between the dispersed phase and dispersion medium, leading to formation of solid microspheres.

a) Emulsification Solvent Evaporation

(c) 2011, JDDT. All Rights Reserved
In the emulsification solvent evaporation method, the organic solvent of the dispersed phase of emulsion is eliminated in two stages.

i) Diffusion of solvent in disperse phase.

ii) Elimination of the solvent at disperse phase-air interface.

\section{b) Emulsification Solvent Extraction Method}

In this technique a continuous phase is used which immediately extract the solvent of the dispersed phase and thus the evaporation stage is no longer

In practice the volume of dispersing phase can be increased by choosing a dispersed phase consisting of cosolvents, of which at least one has great affinity for the dispersing phase. The dispersing phase with two solvents, in which one acts as a solvent extractor of dispersed phase may be used.

\section{Spray Drying Technique}

The principle of spray drying rests on the atomization of a solution (containing the product to be dried) by compressed air or nitrogen through a desiccating chamber and drying across a current of warm air.

It's an established comparatively low cost encapsulation technology that continues to develop. This technique is used to protect sensitive substances against oxidation (e.g. essential oils, vitamins, colorants, etc.). The matrix systems of microsphere type are formed from liquid mixtures comprising an active ingredient dispersed with a polymer inorganic solvent and the resulting dispersion is fed as droplets into heated chamber of spray drier. After spray drying a free flowing product is obtained. 


\section{Fluidized Bed Coating}

The principle of fluidized bed coating or Wurster process is that, solid particles are suspended in the stream of air and a solution of the coating agent or wall forming agent is then sprayed on to the particles.

\section{Centrifugal Extrusión Techniq}

In case of centrifugal extrusion, the core and shell material, and two mutually immiscible liquids are pumped through a spinning two fluids nozzle. This produces a continuous two fluid column, which breaks up into a stream of spherical droplets which are converted to spheres as they fall away from nozzle or are gelled rapidly by collecting in a gelling bath.

\section{Rotational Suspension Separation}

In this process core material dispersed in liquid shell formulation is fed to a rotating disk. Individual core particles coated with a film of shell formulation are flung off the edge of rotating disk along with droplets of pure coating material. When shell formulation is solidified by cooling, discrete microspheres are produced. The technology is claimed to be fast and economic method of encapsulating a variety of materials, which are solids.

\section{Melt Dispersion Technique (Congealable Disperse Phase Encapsulation Procedure)}

The drug is dissolved/ dispersed in the molten lipid/wax under continuous stirring to form a homogeneous blend. During the emulsion step of microsphere preparation, the temperature is maintained at about $10^{\circ} \mathrm{C}$ above the melting point of lipid/wax. A dispersant solution, previously heated to $5^{\circ} \mathrm{C}$ above the lipid/wax melting point, is added to the melt with constant stirring to form an $\mathrm{o} / \mathrm{w}$ emulsion. Hardening of the oily internal phase (containing lipid/wax and drug) and formation of microspheres is accomplished by pouring twice the emulsion volume of ice-cold water $\left(4^{\circ} \mathrm{C}\right)$ into the emulsion.

\section{Spray Congealing Method}

Spray congealing method is a commercial method of forming microspheres. Transition of melt from soft or fluid state to solid state is called as congealing. In this technique, core material is dispersed in a liquefied coating substance and spraying or introducing core-coating mixture into some environmental condition, whereby rapid solidification of coating is occurred. Coating solidification in spray congealing accomplished by thermally congealing molten coating material or introducing coating-core material mixture into a non-solvent.

\section{BIOADHESIVE MICROSPHERES AS ORAL DRUG DELIVERY ${ }^{2}$}

Oral drug delivery is gaining acceptance in terms of improving existing concepts and development of new technologies in the past few years. There is always significant interest in the development of delivery systems via the oral route due to patient compliance and acceptability. During past decade, gastric retention has received much attention with the purpose of maximizing the residence of the dosage vehicle in the stomach, thus solving a specific absorption window issue or for localized drug delivery.
Several approaches to extend the gastric residence time have been investigated including intra-gastric floating devices, bioadhesive technology, high-density system, magnetic devices, unfoldable, expandable or swellable drug delivery systems and super porous hydrogels.

One of the programs associated with gastric retention is the bioadhesive concept, whereby gastric retention can be achieved by adhering drug-loaded particles coated with specific polymers to the wall of the luminal lining (i.e., the mucus layer) with sufficient strength to resist gastric emptying.

Oral delivery is the simplest way of drug administration. In oral drug delivery, the microspheres have to pass through frequently changing environment in the GIT. There is also patient-to-patient variation in GI content, stomach emptying time and peristaltic activity, although constraints of the oral route are numerous, on the whole, it offers less potential danger than the parenteral route.

The relatively brief transit time of about $12 \mathrm{~h}$ through the GI tract limits the duration of action that can be expected via the oral route. Recently, microspheres made from polymers with bioadhesive properties get attached to the stomach and prolong the residence time in the stomach.

Bioavailability of drug with limited solubility in the stomach or intestine and small absorption rate constant can be improved by increasing the retention time in the stomach. Bioadhesive drug delivery system interacts with the mucus which is a highly hydrated, viscous anionic hydrogel layer protecting the mucosa. The basic structural and functional unit of mucus is called as "mucin". The mucin is composed largely flexible glycoprotein chains, which are cross-linked.

Interest in controlled and sustained drug delivery has been increased considerably during the past decade. It is now possible to employ fairly sophisticated systems, which are capable of performing excellent drug release control. The regulating insulin delivery system using bioadhesive drug delivery is an illustrative example (United States Patent No. 6,063). However for oral administration, these systems are facing limitations to some extent because of the gastrointestinal transit. The duration of most of the sustained release product is approximately $8-12 \mathrm{~h}$ due to short G.I. transit time. Hence, it is necessary to localize the drug delivery systems in selected regions of body over extended period of time.

Successful development of an oral controlled release delivery system is hampered by the wide fluctuations in its GI residence time. Different approaches for prolonging GI residence time include the use of...:

- Multiunit dosage form

- Intra-gastric floating drug delivery system (IGF)

- Sandwich - type polymeric delivery system

- Density based dosage forms

- Particle size relevant to stomach retropulsion

- Bioadhesive delivery system 
One of the approaches of gastro retention is to employ bioadhesive polymer that adhere to mucin /epithelial cell surface.

\section{BIOADHESION ${ }^{3}$}

The term bioadhesion is defined as the attachment of synthetic or natural macromolecules to mucus and/or epithelial surface. Bioadhesive drug delivery systems are delivery systems, which utilize the property of bioadhesion of certain polymers, which become adhesive on hydration and hence, can be used for targeting of a drug to a particular region of the body for extended periods of time. Bioadhesion is an interfacial phenomenon in which the two materials, at least one of which is biological, are held together by means of interfacial forces an artificial material and biological substrate, such as adhesion between a polymer and a biological membrane. In the case of polymer attached to mucin layer of a mucosal tissue, the term "bioadhesion" is used. The mucosal layer lies in number of regions of the body including gastrointestinal tract, urinogenital tract, ear, nose and eye. These mucosal layers represent potential sites for attachment of any bioadhesive system. Bioadhesive drug delivery approach to decrease the rate of GI transit of a drug is based on employing polymer that bind to the GI mucin of epithelial cell surfaces and are thus retained in the stomach or intestine for extended periods of time. Bioadhesive polymers would be useful when applied to any mucus, and perhaps non-mucus, membranes as well by increasing the intimacy and duration of contact of a drug with absorbing membrane.

Adhesion can be defined as the bond produced by contact between a pressure sensitive adhesive and a surface. American Society of Testing and Materials, 1984 has defined it as the state in which two surfaces are held together by interfacial forces which may consist of valence forces inter locking action or both.

It is also defined as the ability of a material (synthetic or biological) to adhere to a biological tissue for an extended period of time.

In biological systems four types of bioadhesion can be distinguished such as;

1. Adhesion of a normal cell on another normal cell,

2. Adhesion of a cell with a foreign substance,

3. Adhesion of a normal cell to a pathological cell,

4. Adhesion of an adhesive to a biological substrate.

Bioadhesive are classified into three types based on observable fact, rather than on the mechanisms of bioadhesion.

\section{A. Type I}

Bioadhesion is characterized by adhesion occurring between biological objects without involvement of artificial materials. Examples are Cell fusion and cell aggregation.

\section{B. Type II}

Bioadhesion can be represented by cell adhesion onto culture dishes or adhesion to a variety of substances including metals, woods and synthetic materials.

\section{Type III}

Bioadhesion can be described as adhesion of artificial substances to biological substrates such as adhesion of polymers to skin or other soft tissues.

The aim of the development of bioadhesives are to duplicate, mimic or improve biological adhesion, which are both durable where required and degradable where necessary and non toxic at all. Bioadhesive drug delivery systems utilize the property of bioadhesion of certain water-soluble polymers which become adhesive on hydration and hence, it can be used for targeting a drug to a particular region of the body for extended periods of time.

The mucosal layer lines a number of regions of the body including the GI tract, the urogenital tract, the ear nose and eye. These represent potential sites for attachment of any bioadhesive system and hence, the bioadhesive drug delivery systems may include the following

1. Buccal delivery system

2. Sublingual delivery system

3. Vaginal delivery system

4. Rectal delivery system

5. Nasal delivery system

6. Ocular delivery system

7. Gastrointestinal delivery system

\section{Use of Bioadhesive Preparations}

The idea of bioadhesion was derived from the need to localize drugs at a certain site in the body. Often, the extent of drug absorption is limited by the residence time of the drug at the absorption site. For example, in ocular drug delivery, less than $2 \mathrm{~min}$. are available for drug absorption after instillation of a drug solution into the eye, since it is removed rapidly by solution drainage and hence the ability to extend contact time to an ocular drug delivery system in front of the eye would undoubtedly improve drug bioavailability. In oral drug delivery, the drug absorption is limited by the GI transit time of the dosage form. Since many drugs are absorbed only from the upper small intestine, localizing oral drug delivery system in the stomach or in the duodenum would significantly improve the extent of drug absorption.

Since most of the routes of drug administration such as ocular, nasal, buccal, respiratory, gastrointestinal, rectal and vaginal are coated with the mucus layer, bioadhesive are expected to increase the residence time. In addition, they provide intimate contact between a dosage form and the absorbing tissue which may result in high drug concentration in a local area and hence high drug flux through the absorbing tissue, furthermore, the intimate contact may increase the total permeability of high molecular weight drugs such as peptides and proteins.

\section{Advantages of Bioadhesive Drug Delivery System ${ }^{4}$}

Some of the advantages of bioadhesive drug delivery system are as follows 
1. It localizes drug in a particular region thereby improving and enhancing bioavailability for those drugs with bioavailability problems.

2. The long interaction between polymer and mucus lining of the tissue helps to increase contact time and permit localization, an essential issue when modification of tissue permeability is important for delivery e.g. peptides/proteins and ionized species.
3. It can be helpful to inhibit metabolizing enzymes in localized area.

4. It delivers agents locally for the purpose of modulating antigenicity.

In most instances, the bioadhesive polymer is in contact with a soft tissue (buccal, intestinal, nasal, etc.) and thus the tissue layer responsible for formation of adhesive interface is mucus 5 .

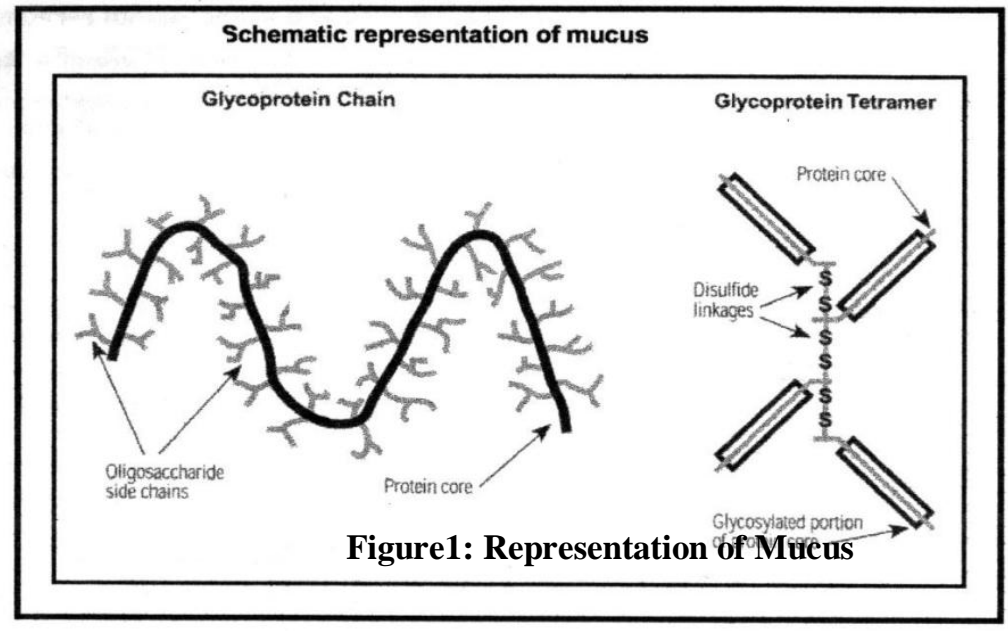

\section{BIOADHESIVE POLYMERS ${ }^{6}$}

These are generally hydrophilic macromolecules that contain the numerous hydrogen bond forming groups (e.g.: hydroxyl and carboxyl groups) and will hydrate and swell when placed in contact with water. In most cases, these materials require wetting to become adhesive. However, over-hydration may result in the formation of slippery mucilage and a loss of adhesive properties

The adhesive bond between a bioadhesive system and a mucus gel can be investigated in terms of the contribution of the following three reasons:

- The surface of bioadhesive polymer.

- The interfacial layer between the bioadhesive material and the mucosa.

- The mucosal surface.

Furthermore, it is reasonable to suggest that an increase in the mechanical strength of the mucus layer by the bioadhesive polymer could result in the strong bioadhesion.

Many types of forces can be used to anchor a polymer to a mucus and /or tissue surface. Covalent forces are suitable, provided the polymeric material is nontoxic to the tissue. More likely polymer candidates will be those that are capable of either weak polar or electrostatic interactions. Undoubtedly, the ultimate force for any polymeric material attached to a tissue will be a combination of forces including hydrophilic and hydrophobic.

It is also clear that strong interaction between chemicals groups on the polymer and mucus/tissue are needed to keep the dosage form in contact with the tissue for an intended period of time.
An ideal polymer for bioadhesive drug delivery should have following characteristics:

1. The polymer and its degradation products should be non-toxic and non-absorbable from gastro-intestinal tract.

2. It should be non-irritant to the mucous membrane.

3. It should preferably form a strong non-covalent bond with the mucin epithelial cell surface.

4. It should adhere quickly to moist tissue and should possess some site specificity.

5. It should possess some sustained release property.

6. It should allow easy incorporation of drug and offer no hindrances to its release.

7. It must not decompose on storage or during life of the dosage form.

8. The cost should not be high; so that the prepared product remains competitive.

\section{Classification of Bioadhesive Polymer ${ }^{7}$}

Many bioadhesives are made up of either synthetic or natural polymers. Most of synthetic bioadhesive polymers are either polyacrylic acid or cellulose derivatives. Examples of polyacrylic acid based polymers are carbopol, polycarbophill, polyacrylic acid (PAA), polyacrylate, poly (methylvinylether-co-methacrylic) acid and poly (methacrylate). Cellulosics include carboxy methylcellulose, sodium carboxy methylcellulose, methylcellulose and hydroxypropyl methylcellulose. In addition (semi) natural bioadhesive polymers include $\mathrm{CH}$ and various gums such as guar, xanthenes, pectin and 
alginate. Finally poly (vinylpyrrolidone) and poly (vinyl alcohol) can be included as synthetic bioadhesive polymers

In more functional type of classification bioadhesive polymers can be grouped into:

- Water soluble polymers, which are typically linear or random e.g. PAA.

- Water insoluble polymers, which are form a swellable network, by covalent or ionic bonds via cross-linking agent e.g. Polycarbophil.

In case of water-soluble polymers, the duration of residence time on tissue surfaces is based on dissolution rate of polymer. In contrast cross- linked polymers have a residence time based on the rate of mucus/tissue turnover due to the lack of solubility in common solvents. Choice of a particular polymer type and perhaps specific polymer will depend on a number of formulation issues as well as patient status.

The development of adhesive dosage forms for controlled drug delivery to or via mucus membranes is of interest with regard to local drug therapy, and the systemic administration of peptide and other drug poorly absorbed from the GIT. The bioadhesive materials have been identified as hydrophilic macromolecules containing numerous hydrogen bond forming groups, particularly carboxyl groups. They become adhesive on hydration so referred as wet adhesive.

\section{Mechanism of Bioadhesion ${ }^{4}$}

A complete understanding of how and why certain macromolecules attach to a mucosal tissue surface is not yet available but certain elements of the process are clear. The bioadhesive must spread over the substrate to initiate the intimate contact and to increase the surface area of contact. Chains of the adhesive can inter-diffuse in to the mucus substrate to create a greater area of contact. Forces of attraction and repulsion develop and for successful bioadhesive, the attractive forces dominate. Each of these steps can be facilitated by the nature of the dosage form and how it is applied. Thus, an increase in applied pressure will contribute to intimate contact by causing viscoelastic deformation at the interface. Moreover, a partially hydrated polymer will be drawn to the substrate surface.

A more complete and comprehensive bioadhesion that predicts the adhesions based on the chemical or physical nature of a particular polymer is not yet available. However, there are four classic theories of bioadhesion.

\section{Electronic Theory}

The adhesive polymer and mucus typically have different electronic characteristics. When these two surfaces come in contact, a double layer of electrical charges forms at the interface and then adhesion develops due to the attractive force from electron transfer across the electrical double layer.

\section{Adsorption Theory}

In the adsorption theory, a bioadhesive polymer adheres to mucus because of secondary surface forces such as Van Der wall's forces, hydrogen bonds, or hydrophobic interactions for a bioadhesive polymer with a carboxyl group. Hydrogen bonding is considered to be the dominant force at the interface. On the other hand, hydrophobic interactions can explain the fact that a bioadhesive may bind to hydrophobic substrate more tightly than to a hydrophilic surface.

\section{Wetting Theory}

Primarily applicable to liquid bioadhesive systems, the wetting theory emphasizes the intimate contact between the adhesive and mucus. Thus a wetted surface is controlled by structural similarity, degree of cross linking of the adhesive polymer or use of a surfactant.

\section{Diffusion Theory}

The chains of the adhesive and the substrate interpenetrate one another to a sufficient depth to create a semi permanent adhesive bond. The penetration rate depends on the diffusion coefficients of both interacting polymer. The diffusion coefficient is known to be dependent on molecular weight and cross linking density. In addition, segment mobility, flexibility of bioadhesive polymer, mucus glycoprotein, and the expanded nature of both networks are important parameters that need to be considered.

These general theories are not particularly useful in establishing of mechanistic pace to modern bioadhesive but they do identify variables that are important to bioadhesion process.

\section{Stages of Bioadhesion ${ }^{3}$}

Bioadhesion is believed to occur in following three stages:

- Wetting.

- Interpenetration.

- Mechanical interlocking between mucin and polymer

Hydrocolloids are believed to adhere to mucosa upon hydration, as the synthetic polymer molecules become more freely mobile and are able to orientate adhesive sites favorably with those of the substrate. As the level of hydration increases, adhesive strength was found to decrease, since bioadhesive bonds become overextended. It is proposed that hydrogen bond forming capacity of the polymer is important in this effect and may emphasize the well-documented bioadhesive properties of polymers possessing numerous carboxyl groups such as Carbopol and Polycarbophil. However, greater the swelling property of the polymer, greater is the ionization, which may lead to a reduction in mechanical strength and concomitant reduction in bioadhesive properties (Jain, 2003). Based on the bioadhesion theories, it may be concluded that the most efficient bioadhesive polymer has physicochemical properties that are closely related to those of the mucus substrate.

\section{Factors Affecting Bioadhesion}

Polymer Related Factors

\section{a) Molecular Weight}

Numerous studies have indicated that at certain molecular weight bioadhesion is at a maximum. The interpenetration of polymer molecules is favorable for low molecular weight whereas entanglements are favored for high molecular weight polymers. The optimum molecular weight for the maximum bioadhesion depends on the type 
of polymers. Their nature dictates the degree of swelling in water, which in turn determines interpenetration of polymer molecules within the mucus. The bioadhesive force increases with the molecular weight of the bioadhesive polymer, up-to 100,000 and beyond this level there is not much effect. To allow chain interpenetration, the polymer molecule must have an adequate length. Size and configuration of the polymers molecule are also important factors. For example with polyethylene oxide adhesive strength increases even up-to molecular weight of $4,000,000$, these polymers are well known to contain molecules of highly linear configuration, which contribute to interpenetration with Dextran, molecules with molecular weights as high as 19,500,000 do not exhibit better bioadhesion that of molecules with a molecular weight of $2,00,000$.

\section{b) Concentration of Active Polymer}

There is optimum concentration of polymer corresponding to the best bioadhesion. In highly concentrated systems, the adhesive strength drops significantly. In fact, in concentrated solutions, the coiled molecules become solvent poor and the chains available for interpenetration are few. This result seems to be of interest only for more or less liquid bioadhesive forms.

\section{c) Flexibility of Polymer Chains}

It is important for interpenetration and enlargement, as water-soluble polymers become cross linked, the mobility of the individual polymer chain decreases. As the cross linking density increases, the effective length of the chain which can penetrate into the mucus layer decreases even further and bioadhesive strength is reduced.

\section{d) Spatial Conformation}

Besides molecular weight or chain length, spatial conformation of molecule is also important. Despite a high molecular weight of 1,95,00,000 for dextrans, they have similar adhesive strength to that of polyethylene glycol with a molecular weight of 2,00,000. The helical conformation of dextran may shield many adhesively active groups, primarily responsible for adhesion, unlike PEG polymers which have a linear conformation.

\section{Environment Related Factors}

\section{a) $\mathrm{pH}$}

$\mathrm{pH}$ was found to have significant effect on bioadhesion as observed in studies of polyacrylic polymers cross linked with $\mathrm{COOH}$ groups. $\mathrm{pH}$ influences the charge on the surface of both mucus and the polymers. Mucus will have a different charge density depending on $\mathrm{pH}$ because of differences in dissociation of functional groups on the carbohydrate moiety and amino acids of the polypeptide backbone.

It was observed that the $\mathrm{pH}$ of the medium was critical for the degree of hydration of highly cross linked polyacrylic acid polymers, increasing between $\mathrm{pH} 4$ and $\mathrm{pH} 5$, continuing to increase lightly at $\mathrm{pH} 6$ and $\mathrm{pH} 7$ and decreasing at more alkaline $\mathrm{pH}$ levels. This behavior was attributed to differences in charge density at different $\mathrm{pH}$.

Polycarbophil showed maximum adhesive strength at $\mathrm{pH}$ 3 , the adhesive strength decreases gradually as the $\mathrm{pH}$ increases up-to 5. Polycarbophil did not show any bioadhesive property above $\mathrm{pH}$ 5. This study was the first systematic investigation of the mechanism of bioadhesion, which clearly showed that the protonated carboxyl groups rather than ionized carboxyl groups react with mucin molecules, presumably by numerous simultaneous hydrogen bonding. At $\mathrm{pH}$ above, polycarbophil swells to a large extent than at $\mathrm{pH} 3$ or below. At high $\mathrm{pH}$, however, the chains are fully extended because of the electrostatic repulsion of carboxylate anions. The polymer chains are also repelled by the negatively charged mucin molecules. It has been also observed that, due to hydrogen bonding between hydroxypropyl cellulose and CP934P, interpolymer complexes form at $\mathrm{pH}$ values below 4.5.

\section{b) Applied Strength}

To place a solid bioadhesive system, it is necessary to apply a defined strength. Whatever the polymer, poly (acrylic acid / Divinyl benzene poly (HEMA) or CP934P, the adhesion strength increases when applied to the tissue contact site can affect the depth of interpenetration. If high pressure is applied for a sufficiently long period of time, polymers become bioadhesive even though they do not have attractive interactions with mucin.

\section{c) Initial Contact Time}

The initial contact time between bioadhesives and the mucus layer determines the extent of swelling and the interpenetration of polymer chains. Along with the initial pressure, the initial contact time can dramatically affect the performance of the system. The bioadhesive strength increases as the initial contact time increases. In case of bioadhesive that need to be polymerized at the application sites, the initial contact time is critical. It is easily controlled when bioadhesive are applied to exposed areas such as eye, nose or mouth. For the application of bioadhesive to the GIT, however, the initial contact time cannot be controlled, which is one of the difficulties in applying bioadhesive to the GIT.

\section{d) Selection of Model Substrate Surface}

The handling and treatment of biological substrates during the testing of bioadhesive is an important factor, since physical and biological changes may occur in the mucus gels or tissues under the experimental conditions. The viability of the biological substrate should be confirmed by examining properties such as permeability, electrophysiology or histology. Such studies may be necessary before and after performing the in vitro test using tissues.

\section{e) Swelling}

This characteristic is related to the polymer itself, and also to its environment. Interpenetration of chains is easier as polymer chains are disentangled and free of interactions. Swelling depends both on polymer concentration and on presence of water. When swelling is too great, a decrease in bioadhesion occurs. Such a phenomenon must not occur too early in order to lead to sufficient action for the bioadhesive system. Its appearance allows easy detachment of the bioadhesive system after the discharge of the active ingredient.

\section{Physiological Variables $^{8}$}

a) Mucin Turnover 
The natural turnover of mucin molecules from the mucus layer is important for at least two reasons. First, the mucin turnover is expected to limit the residence time of the bioadhesive on the mucus layer. No matter, how high the bioadhesive strength, bioadhesive are detached from the surface due to mucin turnover. The turnover rate may be different in the presence of bioadhesive; however no information is available on this aspect so far. Secondly, mucin turnover results in substantial amount of soluble mucin molecules. These molecules interact with bioadhesive before they have a chance to interact with the mucus layer. Surface fouling is unfavorable for bioadhesion to the tissue surface. Mucin turnover may depend on other factors such as the presence of food. The gastric mucosa accumulates secreted mucin on the luminal surface of the tissue during the early stages of the fasting. The accumulated mucin is subsequently released by freshly secreted acid or simply by the passage of ingested food, the exact turnover rate of the mucus layer remains to be determinedcalculated a mucin turnover time of 47-200 min. The ciliated cells in the nasal cavity are known to transport the mucus to the throat at a rate of $5 \mathrm{~mm} / \mathrm{min}$. The mucociliary clearance in the tracheal region has been found to be in the range of $4-10 \mathrm{~mm} / \mathrm{min}$.

\section{b) Disease States}

The physiochemical properties of the mucus are known to change during disease conditions such as common cold, gastric ulcers, ulcerative colitis, cystic fibrosis, bacterial and fungal infections of the female reproductive tract and inflammatory conditions of the eye. The exact structural changes taking place in mucus under these conditions are not clearly understood. If bioadhesive are to be used in the disease state, the bioadhesive property needs to be evaluated under the same conditions.

\section{EVALUATION OF BIOADHESIVE MICROSPHERES}

The best approach to evaluate bioadhesive microspheres is to evaluate the effectiveness of bioadhesive polymer to prolong the residence time of drug at the site of absorption, thereby increasing absorption and bioavailability of the drug. The methods used to evaluate bioadhesive microspheres include the following.

\section{Measurement of Adhesive Strength / in-Vitro Tests ${ }^{9}$}

The quantification of the bioadhesive forces between polymeric microspheres and the mucosal tissue is a useful indicator for evaluating the bioadhesive strength of microspheres. In- vitro techniques have been used to test the polymeric microspheres against a variety of synthetic and biological tissue samples, such as synthetic and natural mucus, frozen and freshly excised tissue, etc. The different in-vitro methods include the following:

\section{a) Tensile Stress Measurement Wilhelmy Plate Technique ${ }^{10}$}

The Wilhelmy plate technique is traditionally used for the measurement of dynamic contact angles and involves the use of a microtensiometer or a microbalance. The CAHN dynamic contact angle analyser (model DCA 322, CAHN instruments, Cerritos) has been modified to perform adhesive microforce measurements. The DCA 322 system consists of an IBM compatible computer and a microbalance assembly). The microbalance unit consists of stationary sample and tare loops and a motor powered translation stage. The instrument measures the bioadhesive force between mucosal tissue and a single microsphere mounted on a small diameter metal wire suspended from the sample loop in microtensiometer The tissue, usually rat jejunum, is mounted within the tissue chamber containing Dulbecco's phosphate buffered saline containing 100 $\mathrm{mg} / \mathrm{dl}$ glucose and maintained at the physiologic temperature. The chamber rests on a mobile platform, which is raised until the tissue comes in contact with the suspended microsphere. The contact is held for $7 \mathrm{~min}$, at which time the mobile stage is lowered and the resulting force of adhesion between the polymer and mucosal tissue is recorded as a plot of the load on microsphere versus mobile stage distance or deformation. The plot of output of the instrument is unique in that it displays both the compressive and the tensile portions of the experiment. By using the CAHN software system, three essential bioadhesive parameters can be analyzed. These include the fracture strength, deformation to failure and work of adhesion.

\section{b) Fracture Strength}

It is the maximum force per unit surface area required to break the adhesive bond.

\section{c) Deformation to Failure}

It is the distance required to move the stage before complete separation occurs. This parameter is dependent on the material stiffness and the intensity of strength of adhesion.

\section{d) Work of Adhesion}

It is a function of both the fracture strength and the deformation to failure. It tends to be the strongest indicator of the bioadhesive potential. This technique allows the measurement of bioadhesive properties of a candidate material in the exact geometry of the proposed microsphere delivery device and the use of a physiological tissue chamber mimics the in-vivo conditions. From a single tensile experiment, 11 bioadhesive parameters can be analyzed out of which 3 are direct predictors of the bioadhesive potential). The CAHN instrument, although a powerful tool has inherent limitations in its measurement technique, makes it better suited for large microspheres (with a diameter of more than $300 \mu \mathrm{m}$ ) adhered to tissue in-vitro.

\section{Novel Electromagnetic Force Transducer $(\text { EMFT) })^{11}$}

The EMFT is a remote sensing instrument that uses a calibrated electromagnet to detach a magnetic loaded polymer microsphere from a tissue sample. It has the unique ability to record remotely and simultaneously the tensile force information as well as high magnification video images of bioadhesive interactions at near physiological conditions. The EMFT measures tissue adhesive forces by monitoring the magnetic force required to exactly oppose the bioadhesive force. To test a microsphere, it must first be attached to the sample of tissue; magnetic force is then generated by an electromagnet mounted on the microscope vertically above the tissue chamber. After the computer has calculated the position of microsphere, the tissue chamber is slowly moved down, away from the magnet tip. As the tissue slowly descends away from the magnet, the video analysis 
continuously calculates the position of microsphere until the latter is completely pulled free of the tissue. The computer can display the results either as raw data or convert it to a force versus displacement graph. The primary advantage of the EMFT is that no physical attachment is required between the force transducer and the microsphere. This makes it possible to perform accurate bioadhesive measurements on the small microspheres, which have been implanted in vivo and then cut (along with the host tissue) for measurement. This technique can also be used to evaluate the bioadhesion of polymers to specific cell types and hence can be used to develop bioadhesive drug delivery systems to targetspecific tissues.

\section{Shear Stress Measurement ${ }^{12}$}

The shear stress measures the force that causes a bioadhesive to slide with respect to the mucus layer in a direction parallel to their plane of contact. Adhesion tests based on the shear stress measurement involve two glass slides coated with polymer and a film of mucus. Mucus forms a thin film between the two polymer coated slides, and the test measures the force required to separate the two surfaces. Mikos and Peppas, 1990 designed the in-vitro method of flow chamber. The flow chamber made of Plexiglass is surrounded by a water jacket to maintain a constant temperature. A polymeric microsphere placed on the surface of a layer of natural mucus is placed in a chamber. A simulated physiologic flow of fluid is introduced in the chamber and movement of microsphere is monitored using video equipment attached to a goniometry, which also monitors the static and dynamic behavior of the microparticle.

\section{Other Tests to Measure the Adhesive Strength}

\section{a) Adhesion Number}

Adhesion number for bioadhesive microspheres is determined as the ratio of the number of particles attached to the substrate to the total number of applied particles, expressed as a percentage. The adhesion strength increases with an increase in the adhesion number.

\section{b) Falling Liquid Film Method ${ }^{13}$}

It is a simple, quantitative in situ method, wherein an excised intestinal segment cut lengthwise, is spread on a plastic flute and positioned at an inclined angle. The suspension of microspheres is allowed to flow down the intestinal strip. Particle concentrations entering the segment from the dilute suspension reservoir and leaving the intestinal segment can be determined with the help of Coulter counter to quantify the steady state fraction of particles adhered to the intestinal mucosa. The percent of particles retained on the tissue is calculated as an index of bioadhesion.

\section{c) Everted Sac Technique ${ }^{14}$}

The everted intestinal sac technique is a passive test for bioadhesion testing and involves polymeric microspheres and a section of the everted intestinal tissue. It is performed using a segment of intestinal tissue excised from the rat, everted, ligated at the ends and filled with saline. It is then introduced into a tube containing a known amount of the microspheres and saline, and agitated while incubating for $30 \mathrm{~min}$. Sac is then removed, microspheres are washed and lyophilized, and the percentage of binding to the sac is calculated from difference in the weight of the residual spheres from the original weight of the microspheres. The advantage of the technique is that no external force is applied to the microspheres being tested; microspheres are freely suspended in buffer solution and made to come in contact with the everted intestinal tissue randomly. The CAHN technique and the everted intestinal sac technique, both predict the strength of bioadhesion in a very similar manner. Santos et al., 1999established a correlation between the two in-vitro bioadhesion assay methods which thereby allows one to confidentially utilize a single bioadhesion assay to scan a variety of bioadhesive polymers.

\section{d) Novel Rheological Approach ${ }^{15}$}

The rheological properties of the bioadhesive interface (i.e. of the hydrated gel) are influenced by the occurrence of interpenetration step in the process of bioadhesion. Chain interlocking, conformational changes and the chemical interaction, which occur between bioadhesive polymer and mucin chains produce changes in the rheological behaviour of the two macromolecular species. The rheological studies provide an acceptable in-vitro model representative of the in-vivo behaviour of bioadhesive polymers Due to intermolecular interactions between the two polymers (mucin and the bioadhesive polymer), experimentally measured viscosity of the mixture is generally higher than the viscosity calculated as a weighted average of the viscosities of the individual components. Thus, the magnitude of the intermolecular interactions can be quantitated by the relative change of the solution viscosity. A synergistic increase in the viscosity of the gastric mucus glycoprotein has been observed with polyacrylates, which thereby reinforce the gastro-duodenal mucus. It has been reported that an optimum polymer concentration is required for rheological synergy to be evident, above which any synergy is masked by the rheological properties of the polymer alone. The effect of $\mathrm{pH}$ on the mucus/polymer rheological synergism of polyacrylates has been examined using dynamic oscillatory rheology. It has been shown that an optimum mucus polymer interaction occurs not only at the $\mathrm{pKa}$ value but also at the $\mathrm{pH}$ regimes unique to each polymer type, being influenced by the hydrogen-bonded interactions.

\section{Measurement of The Residence Time / in-Vivo Techniques}

Measurements of the residence time of bioadhesives at the application site provide quantitative information on their bioadhesive properties. The GIT transit time of many bioadhesive preparations have been examined using radioisotopes and the fluorescent labeling techniques.

\section{GI Transit Using Radio-Opaque Microspheres ${ }^{16}$}

It is a simple procedure involving the use of radio-opaque markers, e.g. barium sulfate, encapsulated in bioadhesive microspheres to determine the effects of bioadhesive polymers on GIT transit time. Faeces collection (using an automated faeces collection machine) and X-ray inspection provide a non-invasive method of monitoring total GIT residence time without affecting normal GIT motility. Bioadhesive labeled with Cr-51, Tc-99m, In-113m, or I123 has been used to study the transit of the microspheres in the GIT. 
Distribution and retention time of the bioadhesive intravaginal microspheres can be studied using the gamma scintigraphy technique. A study has reported the intensity and distribution of radioactivity in the genital tract after administration of technetium labelled HYAFF microspheres. Dimensions of the vaginal cavity of the sheep can be outlined and imaged using labelled gellan gum and the data collected is subsequently used to compare the distribution of radiolabelled HYAFF formulations. The retention of bioadhesive-radiolabelled microspheres based on HYAFF polymer was found to be more for the dry powder formulation than for the pessary formulation after $12 \mathrm{~h}$ of administration to vaginal epithelium. The combination of sheep model and gamma scintigraphy method has been proved to be an extremely useful tool for evaluating the distribution, spreading and clearance of vaginally administered Bioadhesive Drug Delivery Systems.

Table 1: Application of Bioadhesive Microspheres

\begin{tabular}{|c|c|c|c|}
\hline Drug & $\begin{array}{c}\text { Route of } \\
\text { administration }\end{array}$ & Polymer(s) Use & Comments \\
\hline Acyclovir & Ocular & $\mathrm{CH}-$ & Slow released rate increased AUC \\
\hline $\begin{array}{c}\text { Methyl } \\
\text { Pednisolole }\end{array}$ & Ocular & Hyaluronic acid & $\begin{array}{l}\text { Slow released rate, sustained drug concentration in } \\
\text { tear fluids. }\end{array}$ \\
\hline Gentamycin & Nasal & DSM + LPC & Increased nasal absorption. \\
\hline Insulin & Nasal & $\mathrm{DSM}+\mathrm{LPC}$ & $\begin{array}{l}\text { Efficient delivery of insulin in to systemic } \\
\text { Circulation via nasal route. }\end{array}$ \\
\hline $\begin{array}{l}\text { Humanm growth } \\
\text { hormones }\end{array}$ & Nasal & $\mathrm{DSM}+\mathrm{LPC}$ & Rapid \& increased absorption. \\
\hline Desmopressin & Nasal & Starch & $\begin{array}{l}\text { Addition of LPC causes five folds increase in Cmax } \\
\text { and to folds increase in Bioavailability. }\end{array}$ \\
\hline $\begin{array}{l}\text { Haemagglutinin } \\
\text { (HA) }\end{array}$ & Nasal & HYAFF & $\begin{array}{l}\text { With mucosal joint: increased serum IgG antibody } \\
\text { response as compare to intramuscular immunization }\end{array}$ \\
\hline Furosemide & GIT & $\begin{array}{l}\text { AD-MMS } \\
\text { (PGEFs) }\end{array}$ & $\begin{array}{l}\text { Increased Bioavailability, Higher AUC effective } \\
\text { absorption from the absorption window. }\end{array}$ \\
\hline Riboflavin & GIT & AD-MMS (PGEFs) & $\begin{array}{l}\text { Increased bioavailability, Higher AUC effective } \\
\text { absorption from the absorption window. }\end{array}$ \\
\hline Amoxicillin & GIT & AD-MMS (PGEFs) & Greater anti $H$. Pylori activity. \\
\hline Vancomycin & Colonic & $\begin{array}{l}\text { PGEF coated with } \\
\text { Eudragit S } 100\end{array}$ & Well absorbed even without absorption enhancers. \\
\hline Insulin & Colonic & $\begin{array}{l}\text { PGEF coated with } \\
\text { Eudragit } S 100\end{array}$ & $\begin{array}{c}\text { Absorbed only in the presence of absorption } \\
\text { Enhancers. E.g. EDTA Salt }\end{array}$ \\
\hline $\begin{array}{l}\text { Nerve growth } \\
\text { factor (NGF) }\end{array}$ & Vaginal & HYAFF & Increased absorption from HYAFF microspheres \\
\hline Salmon Calcitonin & Vaginal & HYAFF & Increased absorption from HYAFF microspheres \\
\hline Pipemidic acid & Vesical & $\begin{array}{c}\text { CMC as muco- } \\
\text { polyssacaride + Edugragit } \\
\text { RL as Matrix polymer. }\end{array}$ & $\begin{array}{c}\text { Enhanced absorption of pipemidic acid though } \\
\text { bioadhesion. }\end{array}$ \\
\hline Indomethacin & Oral & $\begin{array}{l}\text { Alginate + Sodium CMC/ } \\
\text { MC/Carbopol/ HPMC }\end{array}$ & Slow release rates \\
\hline Glipitizide & Oral & $\begin{array}{l}\text { Alginate + sodium CMC/ } \\
\text { MC/Carbopol /HPMC }\end{array}$ & Slow release rates \\
\hline
\end{tabular}

AD-MMS: adhesive micromatrix system; AUC: area under curve; CMC: carboxy methyl cellulose: DSM: degradable starch microspheres; EDTA: ethylene diaminetetraacetic acid; GIT ; gastrointestinal tract; HYAFF : hyaluronic acid esters ; IgG : immunoglobulin G; IM. : intramuscular; LPC:lysophatidylcholine ; MRT : mean residence time; PGEFs : polyglycerol esters of fatty acids ; MC : methyl cellulose ; HPMC : hydroxypropyl methylcellulose.

\section{CONCLUSION}

Bioadhesive microspheres offer unique carrier system for many pharmaceuticals and can be tailored to adhere to any mucosal tissue, including those found in eyes, oral cavity and throughout the respiratory, urinary and gastrointestinal tract. The bioadhesive microspheres can be used not only for controlled release but also for targeted delivery of the drugs to specific sites in body. Polymeric science needs to be explored to find newer bioadhesive polymers with the added attributes of being biodegradable, biocompatible, bioadhesive for specific cells or mucosa and which could also function as enzyme inhibitors for the successful delivery of proteins and peptides. 


\section{REFERENCES}

1. Benoit JR, Marchais H, Rolland H, Velde VV. Biodegradable Microspheres, Advances in Production Technology. In: Microencapsulation, Methods and Industrial Applications, Benita, S. (Ed.), Marcel Dekker, New York: 1996. Vol. 73, P. 35-72.

2. Singh M, Ind. J. Pharm. Sci., 2002, 5, 1-2.

3. Jain, N. K. Controlled and Novel Drug Delivery. New Delhi: CBS publishers; 2003. P. 353-381.

4. Nagai T, Machida Y, Pharm. Int., 1985, 6, 196-200

5. Allen A, Structure and Function of Gastrointestinal Mucus. In: Physiology of the Gastroenterology Tract, Johnson L. (Ed.), $1^{\text {st }}$ ed. New York: Raven Press; 1981. P. 617-639.

6. Beyer TA, Rearick JJ, Paulson JC, Prieels JP, Sadler JE, Hill RL, J. Biol. Chem., 1979, 254, 125-31.

7. Lee JW, J. Pharm. Sci., 2000, 89(7), 850-866.

8. Lehr CM, Biomaterials, 1992, 18, 4327-32.

9. Mathiowitz E, Chickering D, Jacob JS, Santos CA. Bioadhesive Drug Delivery Systems. In: Mathiowitz, E. (Eds.), Encyclopedia of Controlled Drug Delivery, New York: Wiley and Sons; 1999. Vol. 1. P. 9-44

10. Santos CA, Jacob JS, Hertzog BA, Freedman BD, J. Control. Rel., 1999, 61, 113-122.
11. Hertzog BA, Mathiowitz E. Novel Magnetic Technique to Measure Bioadhesion. In: Bioadhesive Drug Delivery Systems Fundamentals, Novel Approaches and Development, Mathiowitz, E., Chickering, D.E., Lehr, C.M. (Eds.), New York: Marcel Dekker; 1999. Vol. 98. P. 147-171.

12. Kamath KR, Park K. Mucosal Adhesive Preparations, In: Encyclopedia of Pharmaceutical Technology, Swarbrick, J, Boylan JC, (Eds.), New York: Marcel Dekker; 1994. Vol. 10. P. 133-163.

13. Teng CLC, Ho NFH, J. Control. Rel., 1987, 6, 133-149

14. Hong Z, Chasan B, Bansil R, Turner B, Bhaskar K, Afdhal N, Biomacromolecules, 2005, 6(6), 3458-66.

15. Riley RG, Smart JD, Tsibouklis J, Dettmar PW, Hampson F, Davis JA, Kelly G, Wilber R.W, Int. J. Pharm., 2001, 217, 87100 .

16. Brahmankar DM, Jaiswal SB. Biopharmaceutics and Pharmacokinetics: A Treaties. $1^{\text {st }}$ ed. New Delhi: Vallabh Prakashan; 1995. P. 335-357.

17. Richardson JL, Whetstone J, Fisher NF, Watts P, Farraj NF, Hinchcliffe M, Benedetti L, Illum L, J. Control. Rel., 1996, 42, 133-142. 